\title{
Research on Monitoring Crowd Density Method Based on GIS and Mobile Location Data
}

\author{
Hao $\mathrm{ZHAO}^{1,{ }^{,},{ }^{*},{ } \mathrm{Yi}-\mathrm{Fan} \text { QIU }}{ }^{2}$ and Wei DENG ${ }^{3}$ \\ ${ }^{1,2,3}$ School of Resource and Environment Engineering, Wuhan University of Technology, Wuhan, China \\ azhaohao_wut@163.com \\ *Corresponding author: Hao ZHAO
}

\begin{abstract}
To effectively control and reduce the occurrence of accidents in public places, where crowds are easily gathered, is the problem of urban management. The paper proposes a method of monitoring population density which based on the combination of GIS and mobile location data. With the secondary-development which based on component GIS, this method can position the mobile location data in order to map the graph model and monitor the area, counting the number, area and crowddensity of monitoring area. We design and implement some system functions such as display warning messages and cartographic visualization to provide decision support for urban population management and control.
\end{abstract}

\section{Introduction}

In recent years, the safety accidents caused by gatherings are common, which have brought bad influence to the stability and development of the society. Therefore, it is particularly important to monitor the crowds, it is not only to estimate the movement of the crowds, but also more important to prevent the happening of the personnel casualty accidents.

At present, the monitoring of the crowd is mainly dependent on the analysis of video images or mobile phone positioning data. Compared to the results of other video estimation method, the improved hybrid Gauss model used by Shen $\mathrm{Na}$ et al is better1. Through the analysis of the characteristics of pixels and texture, Ren Qingyun3 and Yue Xiaojuan2 divided the crowd density into several degrees such as dense, denser and crowded to implement monitoring. But the scope of video surveillance is limited, high image resolution is required and it's difficult to recognize the dark image. Baidu uses mobile location data to generate the heat map 7 which divide the population density into several density degrees, but clients cannot get alert and the specific density from it. Martin Wirz et al get continuous positioning data by mobile app8, verify the relationship between walking speed and population density, but the method is only an analysis of the data, cannot display warnings.

In this paper, with the combination of the advantages of mobile location data-low cost, large sample size, wide coverage, strong real-time performance and so on6,11. Using powerful spatial analysis, data processing and other functions of GIS, we divide public space into several monitoring areas. It calculate the crowd density according to monitoring areas and the number of location data, and display warnings. The experiment results show that the system can display warning messages according to the threshold crowd density and highlight the crowded area. This will help the relevant departments grasp the aggregation of the population changes and trends in time, and provide data support for developing contingency plans and taking emergency measures to avoid the occurrence of such public safety incidents.

\section{Crowd Density Estimation Method}

Crowd density estimation method which based on GIS and mobile location data includes extracting monitoring areas, counting the number of people, determining the threshold crowd density and displaying warning messages. 


\subsection{Extracting Monitoring Areas}

In the traditional methods, video images is divided into a number of sub areas to monitor which is according to the proportion of visual9. Another method is generating the monitoring areas based on kernel density analysis automatically. The first method needs a large number of video images to reflect the overall situation of crowd. The second method lacks the objectivity. This paper uses remote sensing image with resolution of $0.3 \mathrm{~m}$ and EPSG:3785projection. After visual interpretation, we extracted monitoring areas.

\subsection{Obtaining Mobile Location Data}

With the development of technology, positioning terminal can be designed into chip size11.Each phone has a positioning function. In big data context, more than $90 \%$ of mobile phone users access the Internet every day. This laid the foundation to access mobile location data. Smart phone location data includes geo-tagged photos, location data and so on4. On the one hand, we can use data mining technique to select and process a large number of network data to obtain location data. On the other hand, mobile locating methods is based on cell phone towers and telephone network. We can negotiate with urban management departments or mobile operators to obtain anonymous location data 10 .

\subsection{Determining the Threshold Crowd Density}

When holdinglarge-scale events, the crowd density will grow rapidly in a limited space. And the probability of accident will increase. Hu Qingmei et al did a research on behavior characters of pedestrian and mechanism of stampede12. She got a conclusion that people prefer to own a $0.27 \mathrm{~m} 2-0.84 \mathrm{~m} 2$ buffer area. The optimum density of crowd is $1.3 \mathrm{~m}-2-1.8 \mathrm{~m}-2$. Ran Lijun et al raised that the maximum crowd density of stampede accident is $9 \mathrm{~m}-2$ according to human physiological size of China5.

Martin Wirzet al evaluated the threshold crowd density of Fuin.J's research findings (Table 1)8. $5.5 \mathrm{~m}^{-2}$ is an alarming value of crowd density. Dangerous crowd forces begin to develop potentially. The stampede accident is probably to occur when the crowd density is between $2 \mathrm{~m}^{-2}$ and $5.5 \mathrm{~m}^{-2} .2 \mathrm{~m}^{-2}$ is a safety value.

Table 1. Chart of crowd density

\begin{tabular}{|c|c|}
\hline Density $\left(\mathrm{m}^{-2}\right)$ & Explains \\
\hline$\leq 2$ & Safety value \\
\hline $2<<5.5$ & Danger value \\
\hline
\end{tabular}

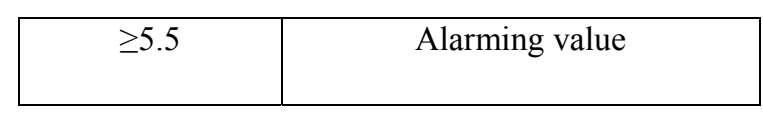

\subsection{Steps of Calculating Crowd Density}

Mobile location data and monitoring areas are combined together based on overlay analysis. Then performing statistical analysis on number of people in every monitoring area.

Calculating the crowd density in every monitoring area. Hence, the density is given as

$$
\rho=\frac{N}{S}
$$

where $N$ is the number of people in monitoring area and $S$ is the area of monitoring area.

Monitoring areas are rendered in different colors according to their density. When reaching the alarming value, it offers warning messages.

\section{Simulation Experiment}

\subsection{Experimental Environment}

The experiment is for testing the method based on GIS and mobile locating data. The experiment used the development methods of GIS which based on C\# language. In a new form, the grading state of crowd density is displayed.

\subsubsection{Simulation of Mobile Location Data}

Based on various researches, the accuracy of mobile phone positioning which based on base station is $\pm 50 \mathrm{~m}$ in the city. In the target zone, some location data are generate randomly. Then these data are superposed a positioning error of $\pm 50 \mathrm{~m}$. The processed data are represented for crowd. 


\subsection{Experiment Process}

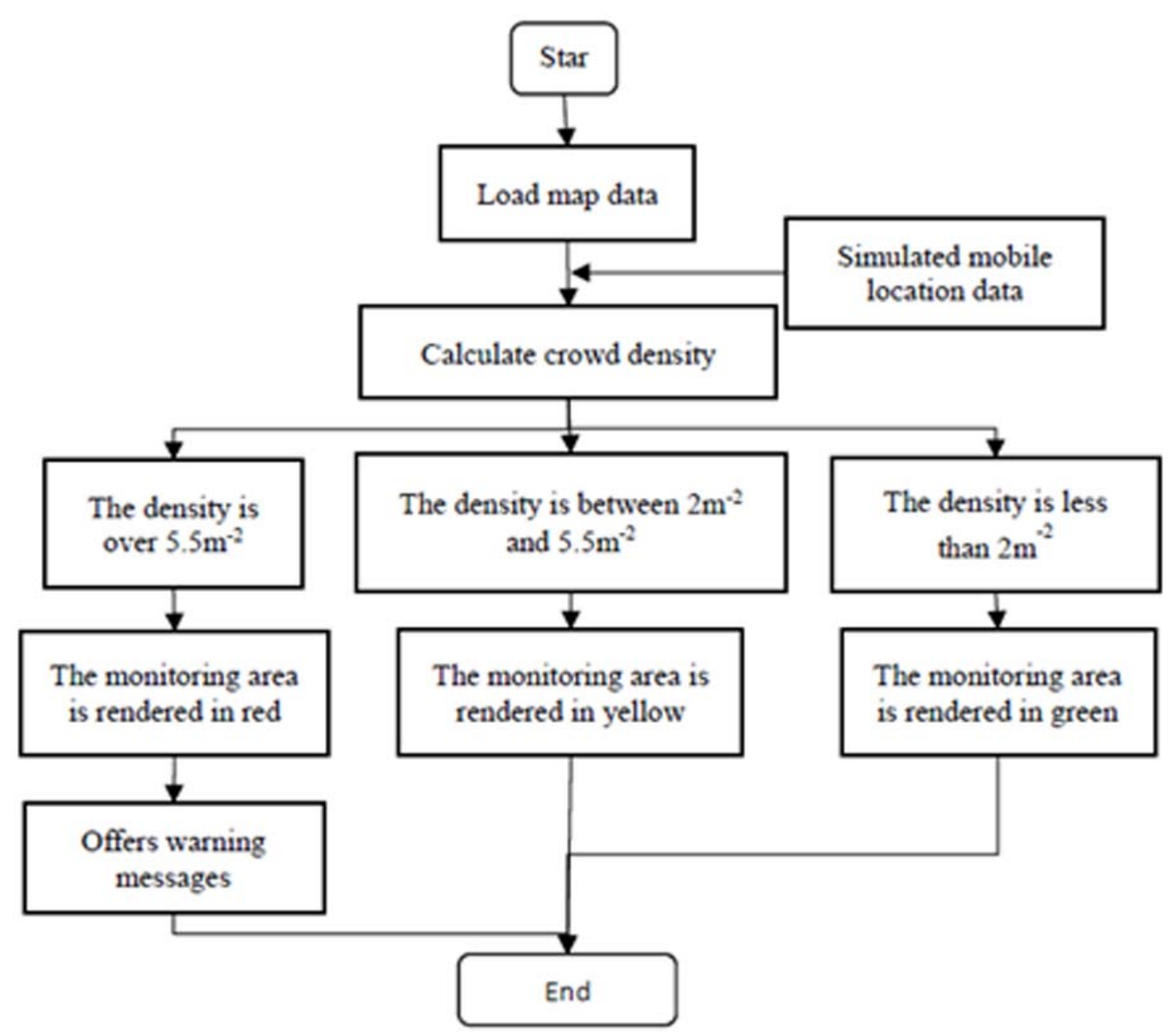

Figure1.Experiment process

\subsection{Analysis of Results}

The crowd density of monitoring area is represented in tabular (Fig 2). It showed the condition of every monitoring area intuitively. Compared to thermodynamic chart, the values of density have more reference value. We can master the degree of congestion from values. Warning messages are displayed in a new form (Fig 3). It can rapidly locate where need management and control. 


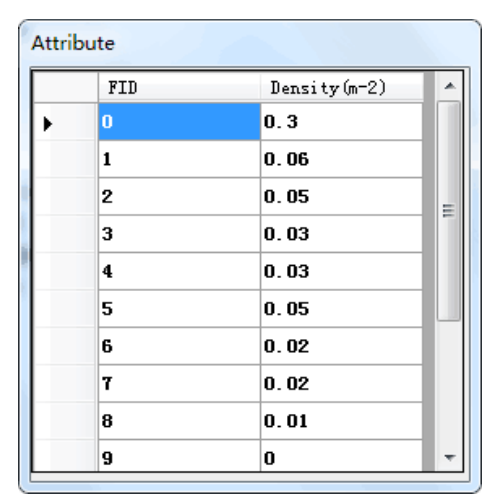

Figure 2. Table of monitoring areas' crowd density

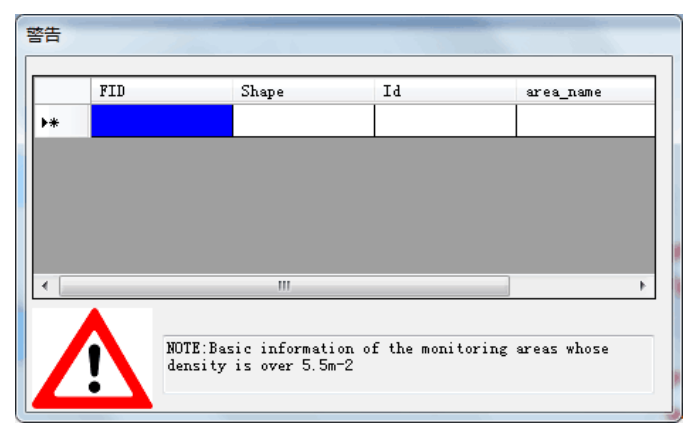

Figure 3.Warning messages

Thermodynamic diagram (Fig 4) showed the crowd distribution. But it was hard to recognize where needmanagement. Some location data were also omitted. Fig5 shows the situation of every monitoring area.

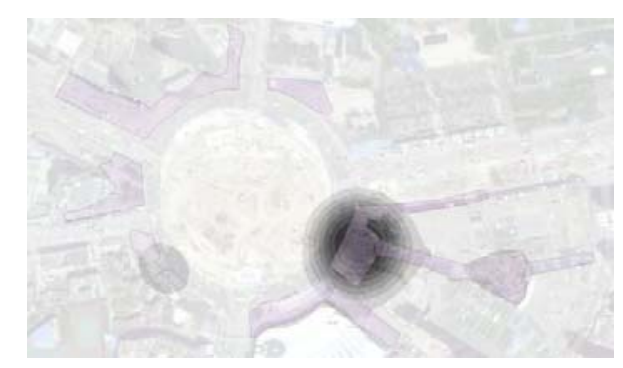

Figure 4. Thermodynamic diagram

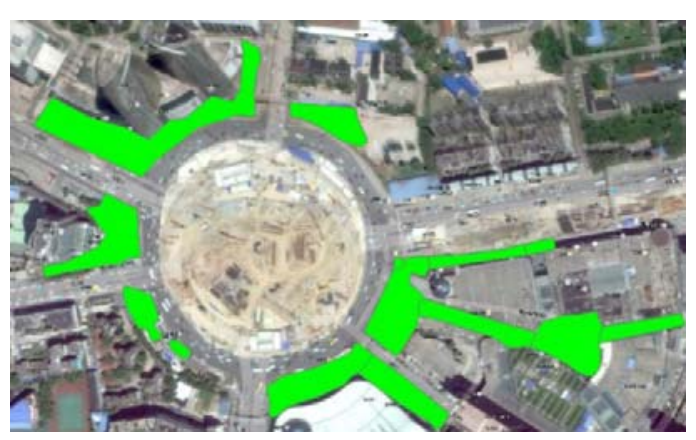

Figure 5. The condition of monitoring areas

\section{Summary}

This paper presents a monitoring crowd density method based GIS and mobile location data. The experiment used simulated mobile location data. The result showed that the method can represent monitoring areas' crowd density. On the basis of monitoring, the system can display warning messages. The timely messages are useful for related stuffs. They can take prearranged plans to prevent the happening of the personnel casualty accidents immediately.

Besides, the mobile location data is applied to the system. So the accuracy of data affects the reliability of monitoring results. To improve the accuracy of monitoring results, how to improve the localization accuracy has become a further research project.

\section{References}

1. Shen Na, Li Ning, Chang Qinglong. Crowd Density Estimation Based on Improved Gaussian Mixture Model. Computer and Digital Engineering, 2012,(7):108-111

2. Yue Xiaojuan. The Algorithm of Crowd Density Detection in Intelligent Monitoring System. Harbin Institute of Technology:2012

3. Ren Qingyun. Crowd Density Estimation methods in Intelligent Video Surveillance.Hebei Normal University: 2011

4. Guo Can, Zhen Feng, Zhu Shoujia. Progress and Prospect of the Application of Smart Phone LBS Data in Urban Researches. Human Geography, 2014, (6):18-23

5. Ran Lijun, Liu Mao. Effects of crowded people density on crushing fatalities. Journal of Safety and Environment,2007,7(4):135-138

6. Lai Jianhui. Research on Data Mining and Analysis in Transportation Based on Mobile Communication Location. Beijing University of Technology:2014

7. Baidu Online Network Technology (Beijing) Co., Ltd.Statistical method and apparatus for passenger flow distribution :China,201010597583.2[P],2011

8. W. Martin, T. Franke, D. Roggen, et al. Probing crowd density through smartphones in city-scale mass gatherings .EPJ Data Science,2013,2:5

9. Zhang Yan, Li Ning, Liu Fumei. A Fast Crowd Density 
Estimation Based on Region Division. Computer and Digital Engineering,2011,(4):128-131

10. Qin Xiao, Zhen Feng, Xiong Lifang, et al. Methods in urban temporal and spatial behavior research in the Big Data Era. PROGRESS IN GEOGRAPHY, 2013, 32(9):1352-1361

11. Chai Yanwei, Zhao Ying, Ma Xiujun, et al. Mobile Positioning Method for Spatial-temporal Behavioral Data Collection and Its Geographical Applications. AREAL RESEARCH AND DEVELOPMENT, 2010,29(6):1-7

12. $\mathrm{Hu}$ Qingmei. Review on Pedestrian Behavior Characteristics and Crowding Mechanism in Public Buildings. China Safety Science Journal, 2008,8(18):69-73 